PAWEŁ PĘDZICH

Department of Cartography

Faculty of Geodesy and Cartography, Warsaw University of Technology

ppedzich@gik.pw.edu.pl

\title{
From cartography of the Universe to molecular cartography - the use of map projections
}

\begin{abstract}
Map projections are very important in the compilation of various types of maps and spatial databases. Geographical information systems provide their users with the significant opportunities in the choice of map projections, coordinate systems, their definitions and transitions between them. The role of map projection can be considered depending on an objective, for which a map has to be used, user of this map and a form of its publication. The Internet, mobile devices and GIS caused that the map projections are used for two main purposes: data visualization and performing of calculations and analyses. The role of map projections is still important, despite the changes occurring in cartography. The rules for the applications of map projections developed over the centuries are still valid. However, the new rules resulting from the new functions of map projections are also created. The aim of this article, that is the author's overview of map projections, is to illustrate the broad spectrum of applications for the map projections.
\end{abstract}

Keywords: map projections, rules for the selection of map projections, mathematical cartography

\section{Introduction}

The history of map projections has been counting for more than two thousand years. The first map projections have appeared in the antiquity yet, in connection with the compilation of the celestial maps. As the years passed, along with the development of different areas of life, the new types of maps, as well as the new map projections, had been being created. During the period of two thousand years hundreds of projections have been elaborated and used in many different ways.

Numerous books and articles have been written about the selective methods of maps, depending on the objectives, for which a map does a good service. Known in Poland and repeatedly cited in the literature, a compilation done by F. Uhorczak (1962) presents the use of map projections to develop the small scale maps. There are a lot of publications on this subject, e.g. the work written by J.P. Snyder (1987). Although, this subject is worth bringing to the readers, especially at the time, when a great deal of maps are compiled increasingly by the persons without sufficient knowledge and education in this field.

\section{Small scale map projections in geographical atlases}

In the case of small scale maps published in geographical atlases, the map projections are selected mainly - but not only - depending on an area shown on a map. For the maps of entire globe the map projections compiled by Aitoff, Hammer, Mollweide are most often used (B. Ogorzelska 2010). J.P. Snyder (1987) also refers to the map projections by Eckert IV and $\mathrm{VI}$, McBryde, Boggs, Robinson, as well as the sinusoidal map projections and cylindrical ones by Mercator and Miller. To present the maps of hemispheres the transverse azimuthal projections by Lambert, Postel, Nicolosi or stereographic and orthographic ones are used. On the maps of the polar regions normal azimuthal projections are used, while for the mapping of the continents conical map projections by Albers, Lambert, Delisle, Ptolemais are most commonly used, as well as pseudoconic one by Bonne, 
azimuthal by Lambert, and stereographic projection in an oblique position (B. Ogorzelska 2010).

\section{Map projections used in navigation}

In the case of navigational maps, traditionally dominated map projections are: the Postel projection, gnomonic projection and Mercator projection. The Postel projection is used in the marine and terrestrial radio communication, as well as for the compilation of tele-communicational maps, due to the keeping of the lengths of lines, which go from the centre point of a map.

In the gnomonic projection all the orthodromes become the straight lines, therefore it is used for planning of long cruises. Because of a large map projection distortion, the large areas of globe should not be represented on the maps of this projection. The Mercator projection due to its conformal properties and linear loxodromic map projection is also used in the navigation. The projection of loxodromes to the straight lines is a convenient property of the projections used in the navigation. One should be aware, that, unfortunately, the length loxodromic distortions within the Mercator projection are very considerable.

An interesting example of the projections preserving the loxodromic lengths and azimuths are the so called loximuthal projections (fig. 1). These are pseudoconic projections - neither conformal nor equal-area ones. The loxodromes, routed from a central point, preserve the lengths and azimuths in these projections.

Another interesting projection, which could be used in the navigation, is the azimuthal two-point projection (fig. 2). It is neither equal-area nor equiangular projection, it has two points, from which all the directions are preserved. It can be used to determine the position of a ship at sea, e.g., if the exact positions of those two radio transmitters and the directions from the ship to the transmitters are known. Other two-point equidistant projection retains the lengths of lines connecting the two selected points on the sphere with any other point of this sphere. This projection can be used to determine the distance to the ship, if its location between the beginning and end of the trip is known.

A number of different map projections has been used in ECDIS (Electronic Chart Display Information System). The plate carrée projection, as well as the Mercator projection, usually

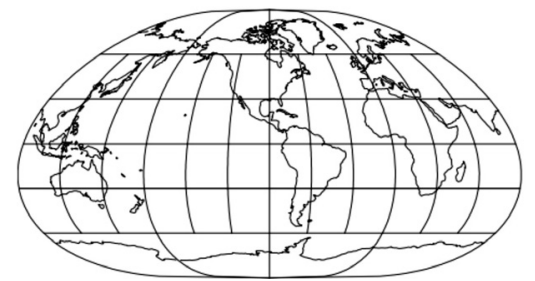

Fig. 1. Loximuthal projection (J.P. Snyder 1987)

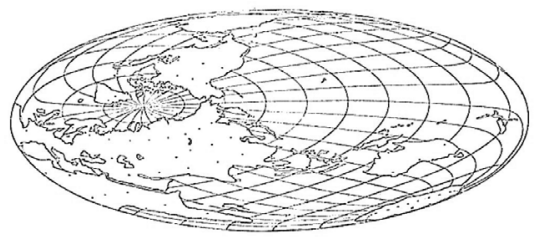

Fig. 2. Two-point azimuthal equidistant projection (https://ktwop.files.wordpress.com/2012/09/ unusual-map-projections-tobler-1999.pdf)

had been being used as the default, i.e. they had been being reverted automatically to a preselected option, for the initial period, but it was not a hard rule. In practice, due to the lack of international standards concerning the use of map projections in ECDIS, the commercial systems provide us with the various choices of map projections (A. Pallikaris 2014).

\section{Projections of topographic and general topographic maps}

The map projection UTM (Universal Transverse Mercator) is a standard for the compilation of topographic maps all over the word. This projection is a modification of the previously developed Gauss-Krüger projection, with the fixed scale of 0.9996 on the axial meridians in the six-degreed projection zones. UTM projection has been used for the development of topographic maps in Poland. Different scale values on the axial meridians depending on the system of coordinates have been applied.

The Lambert projection, which is a conical equiangular projection, is used at today's general topographic maps at the scales of $1: 500,000$ and $1: 1,000,000$. This projection is the basis for the systems of coordinates PL-LCC 
in Poland. It is also used by many other countries to develop the navigation aeronautical charts. On the other hand, the azimuthal stereographic projection is used as a standard for the compilation of topographic maps of the polar areas. It has been used in the armies of NATO as the basis for the construction of GRID's Registration UPS (Universal Polar Stereographic).

\section{Map projections used in the spatial analysis and GIS}

The map projections have a dual role in the geographic informational systems. They are used to compile the maps presenting the results of spatial analysis and to perform the calculations and analysis.

Seeing that the contemporary GIS software allows for the relatively easy transformation of map projections, so, any user, more or less consciously, can compile his or her own map in a map projection freely chosen by him/herself.

According to M. Maher (2010), it is a user, who decides about a choice of map projection for a project, which is performing by him/her. The author gives the general principles and criteria for the selection of map projections from GIS, depending on a scale of a map, as well as a size and location of the geographical regions.

While compiling the maps, presenting the results of spatial analysis in relation to the certain units, e.g., the administrative units, the equal-area map projections are used. For the transport charts, on the basis of which the distances to a certain place, e.g., to the head office of a transportation company, are calculated, the oblique azimuthal equidistant projection can be used.

While identifying and choosing the map projections from GIS, a problem connected with the lack of the uniform terminology very often appears, that is why an attempt towards the standardization of this terminology has made. It has been used, among others, the coding system called the European Petroleum Survey Group (EPSG). EPSG is the scientific organization associated with the European fuel industry, including the professionals from the fields of geodesy, surveying and cartography, working in the search for oil. The EPSG Geodetic Parameter Set, a database, which contains the information about the parameters, that define the shape of the Earth, ellipsoidal reference systems, systems of geographical coordinates, map projections, measurement units etc., has compiled by this organization (http://www.gis-support.pl/co-to-jest-epsg/).

\section{Map projections in web mapping}

The online maps can be compiled in various projections, depending on the purpose and destination, but the projection called WebMercator, which is a modification of the Mercator projection and is the standard in the online mapping applications, such as Google Maps, Bing Maps, MapQuest, deserves our special attention. This map projection is used in the spherical form, even while projecting the ellipsoid, which means, that it is not strict a conformal and the loxodromes do not reproduce themselves exactly into the straight lines. Due to the simple mathematical formulas, it offers the possibility of high-speed computing. Since the poles are projected at the infinity, a map of the whole world in the WebMercator projection is limited to a strip between the parallels of the latitudes $\pm 85.051129^{\circ}$. This map projection is an inappropriate one for the compilation of a map, which depicts the whole globe or the areas sized as the continents or large countries.

In order to avoid these disadvantages, B. Jenny (2012) proposed to use the so-called adapting map projections. A type of projection and its parameters are selected dynamically, so as to obtain the geometry of all map changes in a "seamless" way, without any visual discontinuities at the time, while a user applies some changes to the scale and to the central meridian of longitude.

\section{Projections of celestial maps and maps of celestial bodies}

The celestial maps can be divided into maps of stars, constellations and galaxies. To compile the maps of hemispheres of the celestial sphere the azimuthal map projections are commonly used (fig. 3). A gnomonic map projection is used to present the movement trajectory of meteorites, while a stereographic one is used to present the groups of galaxies. A conical projection is used very often, because of its low distortion. 


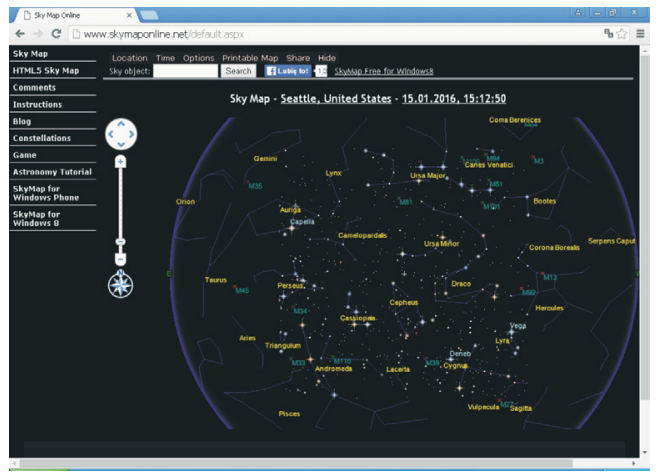

Fig. 3. Celestial map compiled in the azimuthal projection

(http://www.skymaponline.net/instructions.html)

The celestial maps are also compiled in order to present the phenomena appearing in the cosmos. The examples are the maps of distribution of the cosmic microwave background radiation. This radiation is a remnant from the earliest stages of evolution of the Universe, it almost homogeneously fills the whole Universe. In 1965 two American astrophysicists, A. Penzias and $\mathrm{R}$. Wilson, when trying a new antenna, detected the existence of the waves lapping it from every direction. In 1989 the first satellite, which was intended for testing of cosmological COBE (Cosmic Background Explorer), was

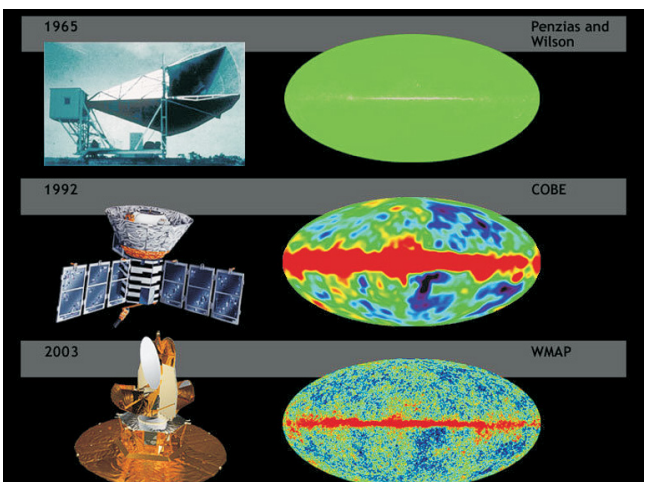

Fig. 4. Maps presenting the results of studies on the relict radiation (https://commons.wikimedia.org/wiki/ File:BigBangNoise.jpg)

launched. It was equipped with an instrument to search for the irregularities in the microwave radiation of the background.

In turn, in 2001 a probe called NASA WMAP (Wilkinson Microwave Anisotropy Probe) was launched with the task of measuring the temperature of the background radiation. The map in figure 4 presents the results of those studies. For their compilation the so-called method of spherical pixilation called HEALPIX (Hierarchical Equal Area iso-Latitude Pixilation) was applied (fig. 5). Pixels are characterized by the equal areas of surface and the distribution of centers
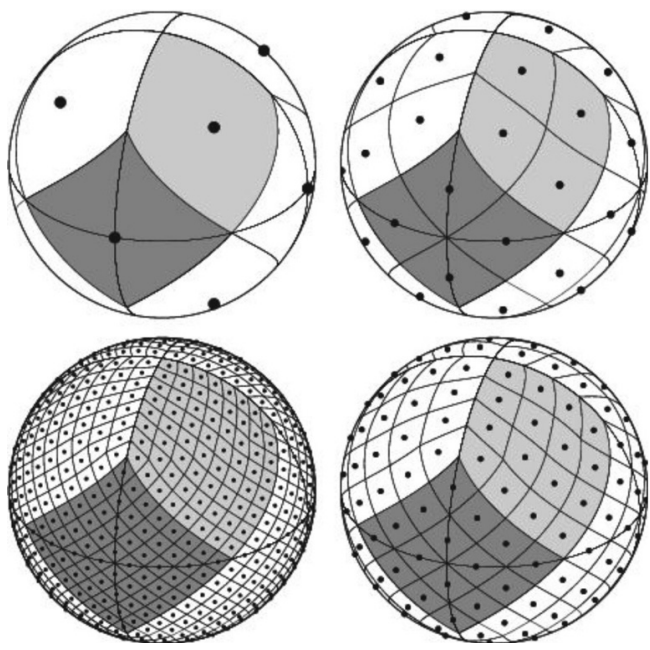

Fig. 5. Pixilation of the HEALPIX Sphere (http://healpix.jpl.nasa.gov/healpixBackgroundPurpose.shtml) 


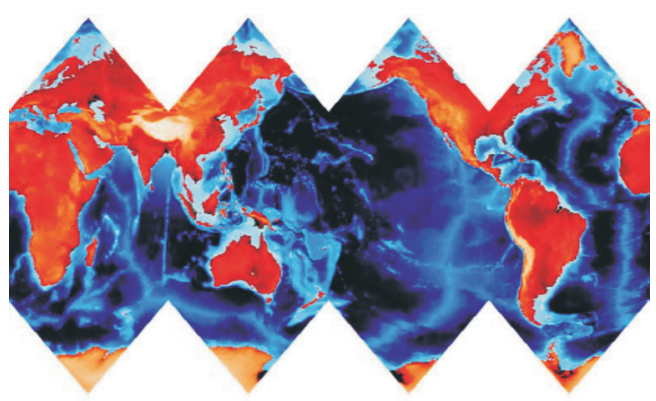

Fig. 6. Map of the Earth in the HEALPIX projection (http://www.das.inpe.br/school/AdvancedCourse/ pdfs/Gorski_lecture.pdf)

with the equal latitudes. The author of this method is K.M. Górski (K.M. Górski and others 1998). The maps, showing the results of the analyses, are carried out mostly in the map projections of Mollweide.

There is also a map projection of the name Healpix, which is composed of the cylindrical projection of circum-equatorial area and zonal Collignon projection for the polar regions (fig. 6).

Another interesting issue in the field of mapping the cosmos is the selection of map projections depicting the extra-terrestrial celestial bodies. This problem is described in many works (among others, P. Pędzich, K. Latuszek 2014). A part of the celestial bodies (large planets) has a shape close to the sphere or ellipsoid, and then, the same projection as for the surface of the Earth, for example, the orthographic projection for image maps depicting the hemispheres of planets, is used.

There are also some objects of an irregular shape, for the mapping of which the non-custom map projections are used, such as the projections of multiaxial ellipsoids or morphographic projections (fig. 7). These letter projections allow us to reconstruct at a plane all the roughness of surface of a celestial body, which is mapped.
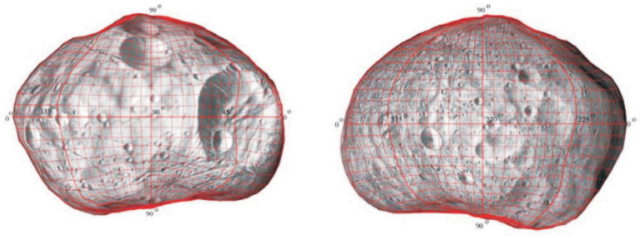

Fig. 7. The morphographic projection - Phobos (one of the moons of Mars) (M. Nyrtsov 2003)

Mathematical formulas of these projections are the functions of three variables: two coordinates, indicating the position of a detail on the surface of a celestial body, and the radius of a celestial body.

\section{Projections of maps and photographs taken from the orbit of the Earth}

A.P. Colvocoress, J.P. Snyder and J.L. Junkins in the years 1973-1979 compiled the space oblique projections for satellite images. We can distinguish two such projections: the Space Oblique Mercator (fig. 8) and the Space Oblique Conic. The first one of them is a modified version of Mercator's cylindrical projection with the display surface defined by the parameters of satellite orbits. It was designed specifically for compiling the maps by combining the satellite images into one continuous image. This projection is recommended for the narrow strips along a track of satellite. The projections for the presentations of satellite tracks are called Satellite-Tracking projections (fig. 9). All the traces of satellites with the same orbital parameters are projected as the straight lines. The cylindrical and conical projections, which are neither conformal nor equal-area, are also used. They were compiled in 1977 by J.P. Snyder (1987).

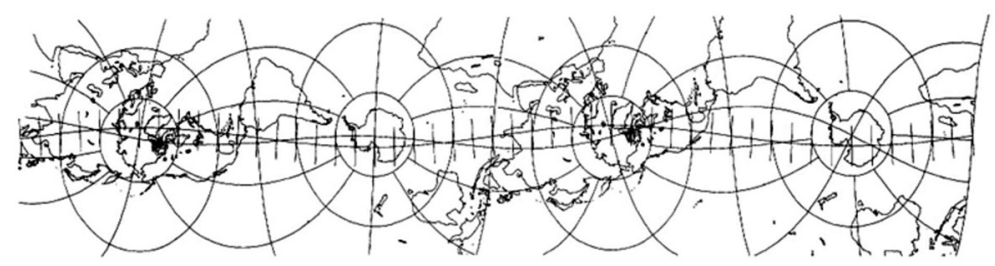

Fig. 8. Space Oblique Mercator projection (J.P. Snyder 1987) 


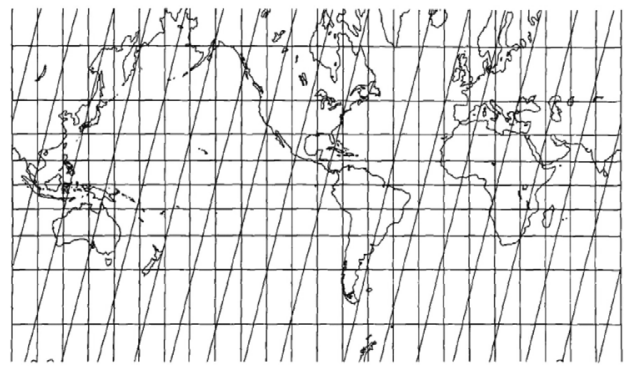

Fig. 9. Cylindrical Satellite-Tracking projection

(J.P. Snyder 1987)

\section{Polyhedral map projection and projections for constructing the globes}

Despite the obvious opportunities to present the Earth's surface in the three-dimensional space, the globes also require to use the map projections for their elaboration. K. Wagner recommended for this purpose the Cassini-Soldner projection (fig. 10), where the globe is divided first into the meridional belts. The sinusoidal and conical projections are also used. The polar regions (above $80^{\circ}$ ) are compiled in the azimuthal stereographic projection or Postel projection (M. Gede 2009). The map projections are also used to develop the pseudo-three-dimensional virtual globes. The models, which are made in the KML format (Keyhole Markup Language), require a series of images in the plate carrée projection, while the models in the VRML format (Virtual Reality Modelling Language) require two different types of map projections: two polar regions should be compiled in the azimuthal equal-distance projection, while the next four images of equatorial regions should be represented in the plate carrée projection. The VRML format is a continuation of the X3D format with the same opportunities to develop the virtual globes (M. Gede 2015).

Regardless of a map projection, in which an output map, used to develop a globe, is compiled, we can see the result in the perspective projections from different outlook. Google Earth and NASA World Wind (fig. 11) show the globe in a way, that it is visible from the space. It is used for this purpose a general perspective projection. If the axis of a camera is set exactly towards the centre of the Earth, we are faced with the vertical perspective, in the other cases it is an tilted perspective projection.

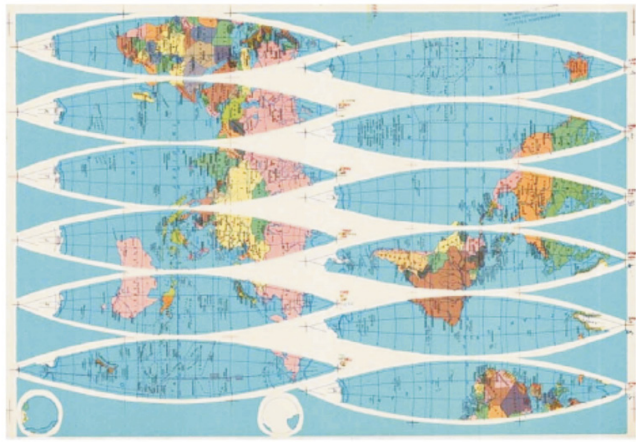

Fig. 10. The stripes for a globe in the Cassini-Soldner projection (M. Gede 2009)

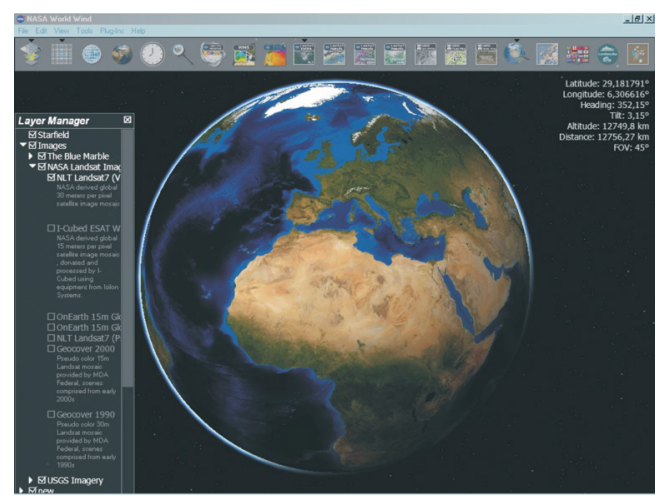

Fig. 11. Virtual Globe World Wind (J.P. Snyder 1987)

The globes in the VRML format present the earthly sphere, which is approximated by a polyhedron (M. Gede 2009). Such solutions have been used in cartography for five centuries. Already in 1525 Albrecht Dürer proposed a projection of the sphere to the polyhedron, as an approximation of the surface of a globe. The polyhedral projections divide the spherical surface on a regular basis, and that is a significant problem for the presentation and data structure within the geographical databases (D. Van Leeuwen, D. Strebe 2006). Figure 12 shows an example of polyhedral projection.

\section{Statistical, propaganda and anamorphic map projections}

The statistical maps are used for the presentations of various phenomena and trends of their 


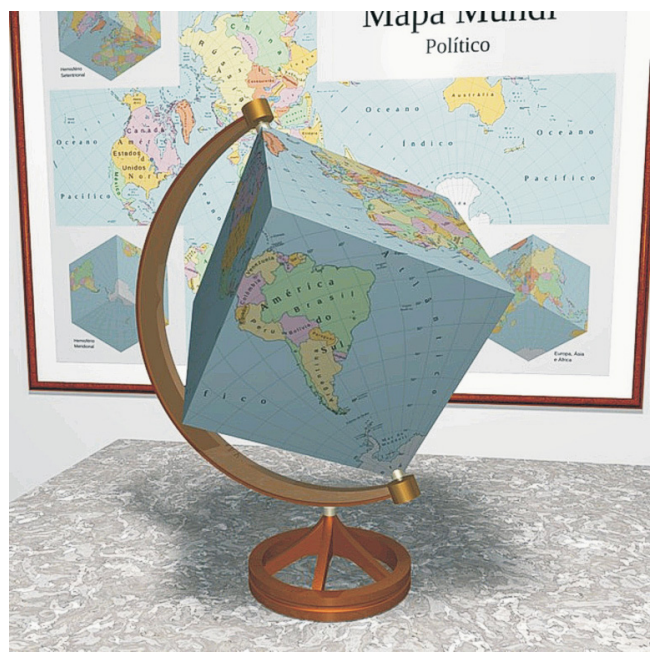

Fig. 12. The Earth in the form of a cube (www.progonos.com/furuti/MapProj/Dither/ProjPoly/ projPoly.html)

changes. For their compilation the methods of presentation relating to the areas of surface are used, for example, among others, the choropleth method. It is obvious then the use of equal-area projections, which help us to maintain the proper relations of areas. For the same reason this type of projections is also applied to the political maps.

Map projections, due to the different distributions of distortions, ideally fit for the propaganda mapping. For instance, the Mercator projection has the little distortions of areas in the immediate vicinity of the equator; these distortions grow quickly with the increasing of latitude. This means, that, as a result, the countries laying in the high geographical latitudes seem to have the disproportionately large areas, than the countries laying in the low geographical latitudes. For example, during the Cold War a presentation of the world on a map in the Mercator projection meant, that the states of the Warsaw Pact occupied on the maps much large area, than it was in the reality; that distortion probably could have an impact on the perception of the political situation in the world (fig. 13).

The map compiled in the Peters projection presents the different aspects and proportions of the same areas (fig. 14). This projection was

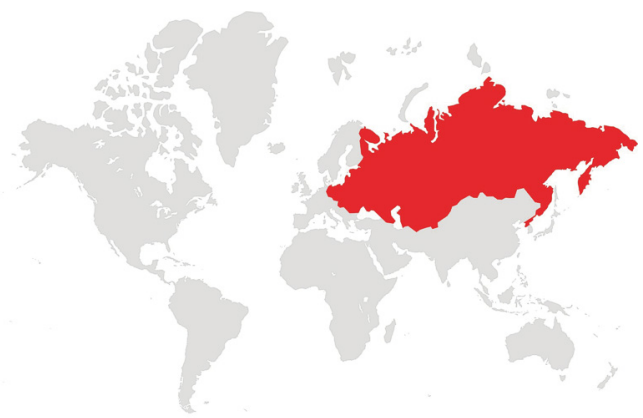

Fig. 13. Map of the world in Mercator projection (http://www.learnnc.org//p/editions/mapping/6434)

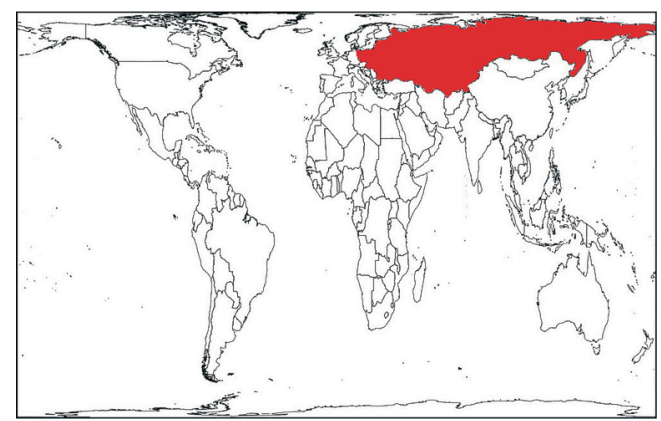

Fig. 14. Map of the world in Peters projection (http://www.learnnc.org/lp/editions/mapping/6434)

introduced in 1972 by A. Peters, as the so-called projection of "social justice". It aroused initially a great opposition from the scientific community of cartographers, but nowadays it is often used on the maps of UNESCO.

The use of the perspective projections also can affect the perception of the political situation. The Western Europe seen from the perspective shown in figure 15 could during the Cold War feel threatened by the Soviet Union.

The so-called anamorphic projections are ideal to compile the propaganda maps. The result of their use can be a large area or highlighted phenomenon on a map.

While studying the migration around the Swedish town of Ashby, T. Hägerstrand in 1957 used the logarithm of length as a radial scale 


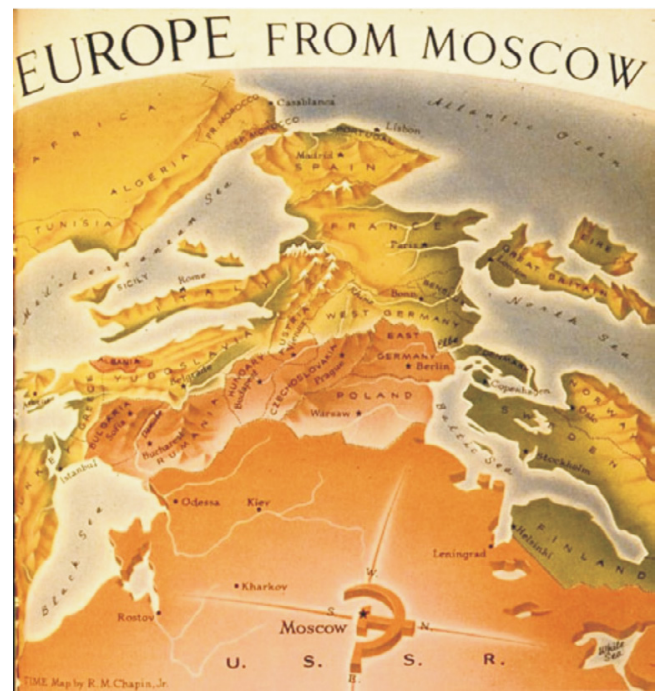

Fig. 15. Map called Europe from Moscow published by R. M. Chapin, Jr. in the journal "Time" March 10, 1952

(http://www.learnnc.org/lp/editions/mapping/6434)

in the projection elaborated by himself. That fact resulted in an increase of the scale in the centre of Asby, near which the majority of migration took place (W. Tobler 2008) (fig. 16).

\section{Map projections used in photography}

The map projections are also used in photography, because the wide-angle photographs

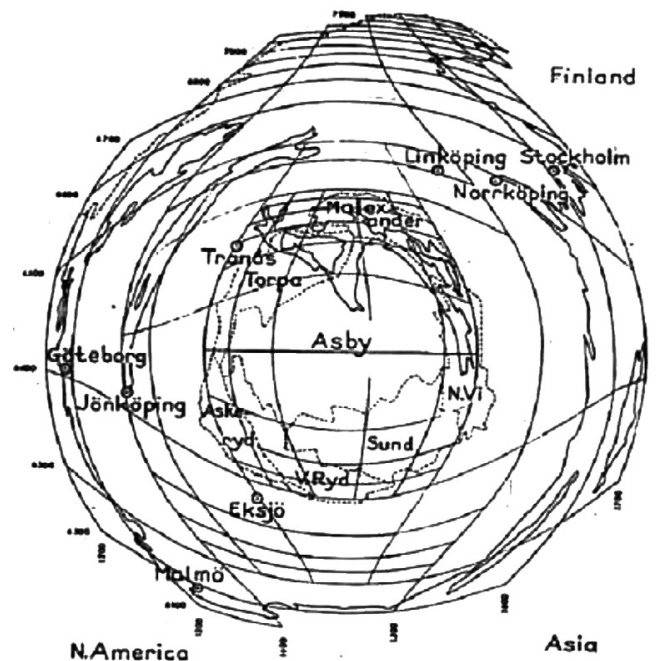

Fig. 16. Map of migration around the Swedish town of Asby in the anamorphic projection

(T. Hägerstrand 1957)

have significant distortions of an image. Visual information seen from a single point is defined on the sphere of view centred at a point of observation. To get a flat image of the sphere, we must project it onto the plane (R. Carroll, M. Agrawala, A. Agarwala 2009).

The discussions about the limitations and possibilities of map projections for the presentation of visual sphere at the plane of image

Perspective

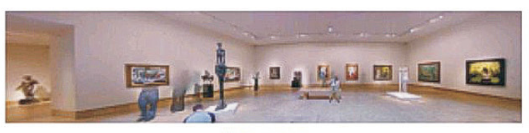

Mercator

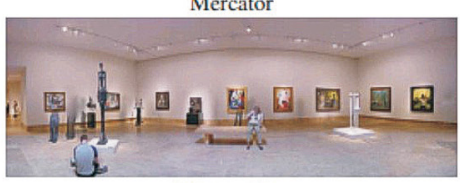

Geographic
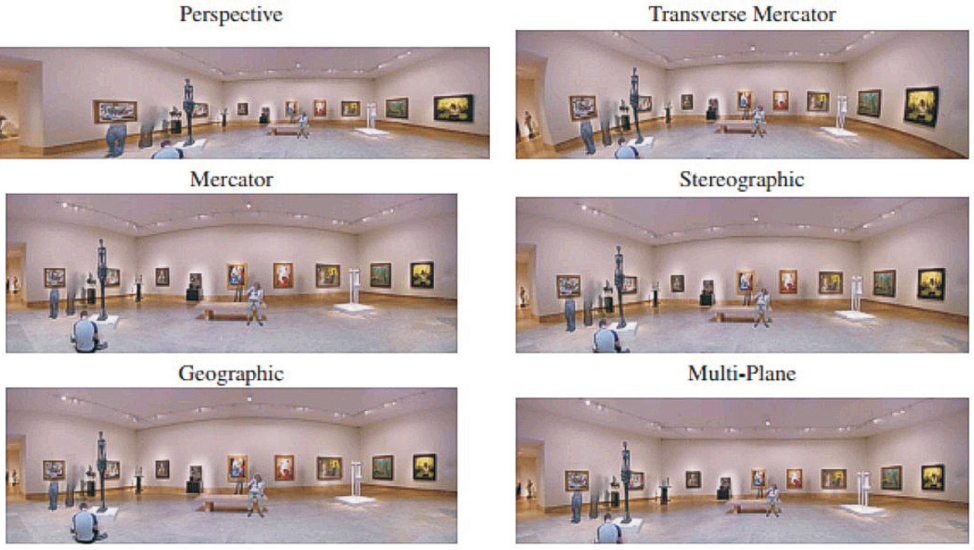

Fig. 17. Panorama in various map projections (L. Zelnik-Manor and others 2005) 


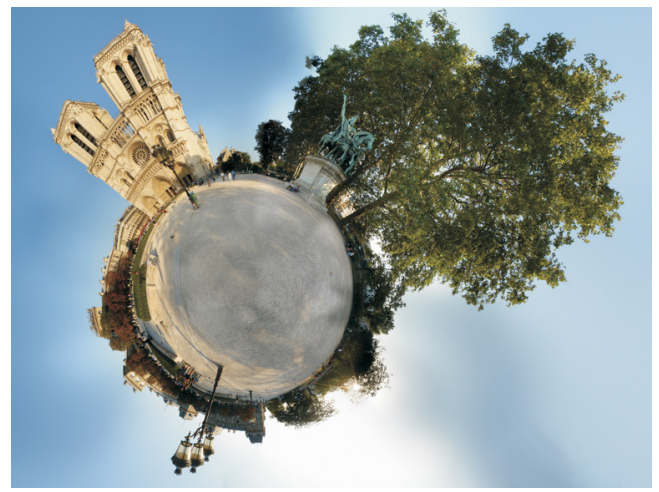

Fig. 18. Spherical Panorama "Globe Panorama" performed by A. Duret-Lutz (https://commons. wikimedia.org/wiki/File:Globe_panorama.jpg\#/ media/File:Globe_panorama.jpg)

were conducted by L. Zelnik-Manor, G. Peters and P. Perona (2005). They had analysed a lot of map projections and their properties, as well as the impact of various parameters of these projections on the final form of an image. They had also proposed a universal solution for the presentation of panoramas and that presentation was the so-called multi-plane projection. Figure 17 presents the results of their research. The most widely known and used in photographic reproduction is the gnomonic projection, but when the field of view approaches the $180^{\circ}$, the distortions will increase here to the infinity. For this reason, the gnomonic projections can be used to image an angle of 40 degrees.

In turn, the Mercator projection can be used for panoramas of $360^{\circ}$, however, to a limited extent for the vertical angle, because when it approaches the $90^{\circ}$, the distortions will grow to the infinity. Thus, the Mercator projection is useful for panoramic images with the large

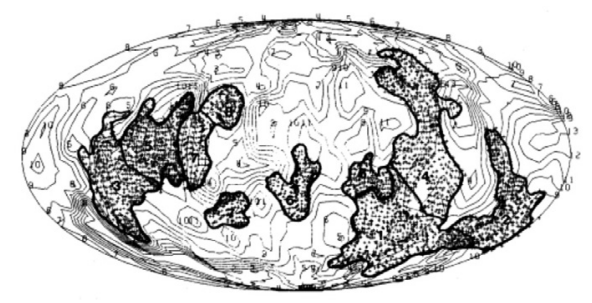

Fig. 19. Mollweide projection for the surface of myoglobin (D. Fanning and others 1986)

horizontal field of view, e.g., for the images of a large group of people (R. Carroll, M. Agrawala, A. Agarwala 2009).

The images of stereographic projection are similar to those made by the lens "Fisheye", as well as to the images with a wide field of view taken in the curvilinear perspective. This projection is used to create the interesting visualizations, which are the spherical panoramas, the kinds of photographs covering the whole surrounding area around a camera - 360 degrees horizontally and 180 degrees vertically (fig. 18).

\section{Map projections used in molecular cartography and crystallography}

Among the applications of map projections one can find the maps of objects with very small dimensions. They are the tools for research in the so-called molecular cartography. This is a method of three-dimensional information on the topography of proteinaceous surface by the contour lines. The contour maps retain the local "topographic" objects and, at the same time, present the global appearance of surface (D. Fanning and others 1986). These maps show
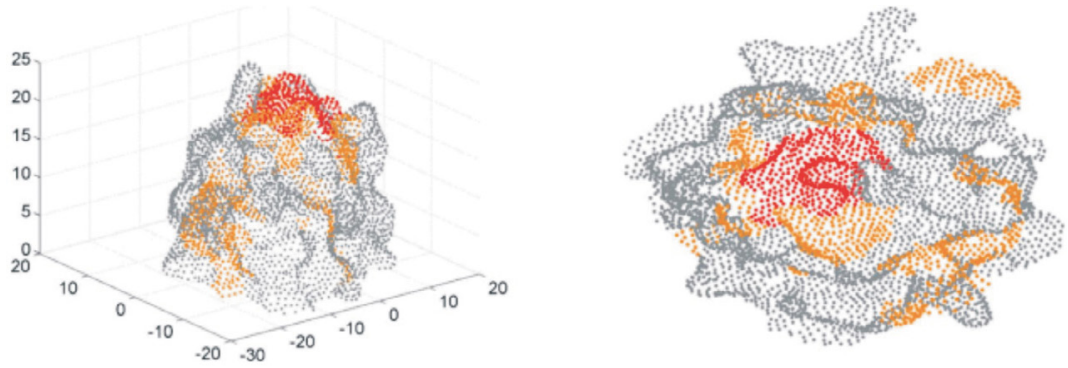

Fig. 20. Maps showing the complex shapes of proteins (H. Yang, R. Qureshi, A. Sacan 2012) 


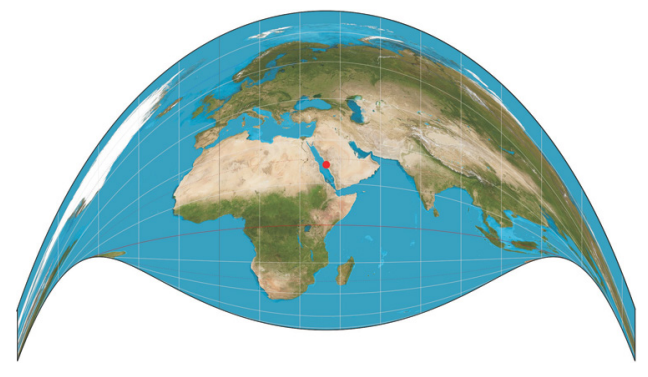

Fig. 21. Retro-azimuthal map projection for Mecca (https://www.mapthematics.com)

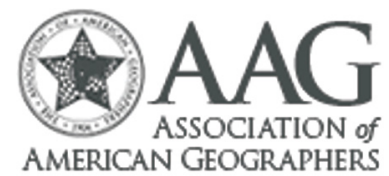

Fig. 22. Logo of the Association of American Geographers
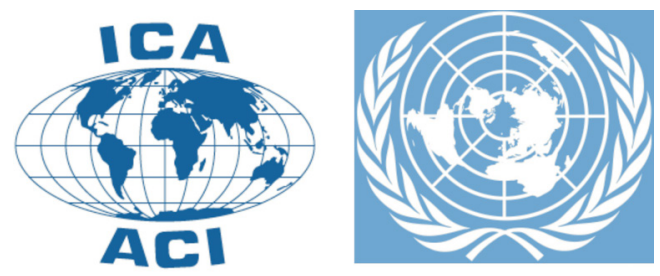

Fig. 23. Map projections in the logos of various organizations the complex structures of proteins at a plane, e.g., the myoglobin. In the first projections of this type the sphere or ellipsoid were taken as the original surfaces (fig. 19). Later, the more complex shapes there also were used $(\mathrm{H}$. Yang, R. Qureshi, A. Sacan 2011) (fig. 20).

The map projections are also used in the crystallography. A stereographic projection was introduced to the crystallography in 1839 by W.H. Miller. It is used for the exact presentation of angles between the normal walls of crystals.

\section{Other projections of interesting properties and applications}

A number of non-standard map projections with specific properties discusses W. Tobler (2008). One of them is the retro-azimuthal projection compiled in 1909. J. Craig presented this type of projection (fig. 21). It keeps the azimuths from any place on the Earth to a central point. For instance, the projection for Mecca keeps the azimuths from any point on the globe in the direction of Mecca. A map in this projection facilitates the followers of Islam a determination of the direction towards Mecca.

In $1879 \mathrm{H}$. Berghaus presented a map of the world in the shape of a star. The projection applied is called the Star projection and used in the decorative purposes, among others, it is used by the Association of American Geographers as the logo of their organisation (fig. 22). The logotypes of other organisations also include the map projections, e.g., in the logo of the United Nations Organisation one can find the Postel projection, while in the logo of the International Cartographic Association the Mollweide
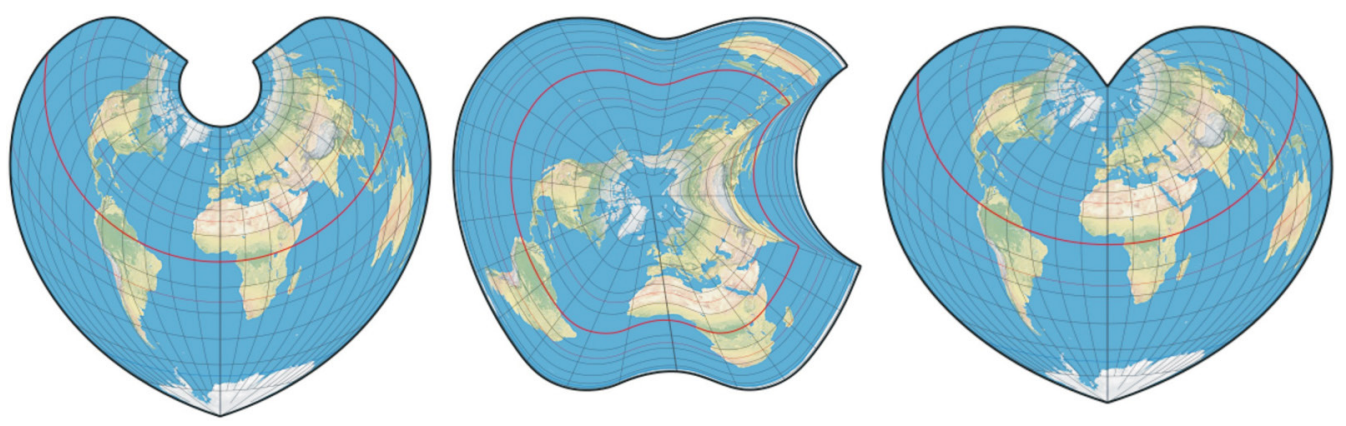

Fig. 24. Map projections for the decorative purposes compiled by D. Strebe (https://www.mapthematics.com) 
projection can be find (fig. 23). Figure 24 shows the examples of cartographic graticules in other decorative projections developed by D. Strebe.

\section{Summary}

The map projections have been presented in cartography for over two thousand years. They are used to compile the maps, as well as to perform the cartometric analysis. The use of the maps, described in this article, shows an important role, which was played by the map projections in the past and is played now, not

\section{Literature}

Carroll R., Agrawala M., Agarwala A., 2009, Optimizing content-preserving projections form wide-angles images. "ACM SIGGRAPH conference proceedings", http://vis.berkeley.edu/papers/capp/projection-sig09.pdf

Fanning D., Smith J., Rose G., 1986, Molecular cartography of globular proteins with application to antigenic sites. "Biopolymers" Vol. 25, pp. 863-883.

Gede M., 2009, The projection aspects of digitizing globes, http://icaci.org/files/documents/ICC_proceedings/ICC2009/html/refer/10_1.pdf

Gede M., 2015, Novel globe publishing techniques using WebGL. "e-perimetron" Vol. 10, no. 2, pp. 87-93.

Górski K.M., Hivon E., Wandelt B.D., 1998, Analysis issues for large CMB data sets. "Proceedings: Evolution of Large Scale Structure", arXiv:astroph /9812350, http://arxiv.org/pdf/astroph/9812350v1.pdf

Jenny B., 2012, Adaptive composite map projections. "IEEE Transactions on Visualization and Computer Graphics" Vol. 18, no. 12, pp. 2575-2582.

Maher, M., 2010, Lining up data in ArcGIS. ESRI Press.

Nyrtsov M., 2003, The classification of projections of irregularly-shaped celestial bodies. In: "Proceedings of XXI International Cartographic Conference", Durban, http://www.cartesia.org/geodoc/ icc2005/ pdf/poster/TEMA23/Nyrtsov\%20Maxim \%20V. Russia.pdf

Nyrtsov M., Bugaevsky L.M., Strooke P., 2007, The multiple axis ellipsoids as reference surfaces for mapping of small celestial bodies. In: "Proceedings of XXIII International Cartographic Conference", Moscow, http://icaci.org/files/documents/ICC_proceedings/lCC2007/html/Proceedings.htm

Ogorzelska B., 2010, Odwzorowania kartograficzne. In: J. Pasławski (ed.), Wprowadzenie do kartografii only in cartography. The knowledge in the scope of map projections accumulated over the years is huge. It is difficult to expect from the contemporary artists and map users a freely movement in the area of knowledge in this scope, particularly, from those really involved in the process of map compilation, but who are not the professional cartographers. Although the principles for the selection of map projections have been known for a long time, we cannot rule out their applications by map users in their sole discretion, especially for the compilation of maps presenting the results of their own research.

i topografii, Wrocław: Wydawnictwo Nowa Era, pp. 81-168.

Pallikaris A., 2014, Choosing suitable map projections for worldwide depiction of electronic charts in ECDIS, http://sssi.org.au/userfiles/docs/Weekly\%20Newsletter/documents_14152302751707881897.pdf

Pędzich P., Latuszek K., 2014, Kartografia planetarna - przykłady opracowań, odwzorowania kartograficzne, nowe wyzwania. "Polski Przegl. Kartogr." T. 46, nr 4, pp. 369-396.

Snyder J.P. 1987, Map projections. A working manual. Washington: United Government Printing Office.

Strebe D., Geocart User's Manual, https://www.mapthematics.com/Downloads/Geocart_Manual.pdf

Tobler W., 2008, Unusual map projections. "Cartographic Perspectives” No. 59, pp. 28-40.

Uhorczak F., 1962, Obraz świata w siatkach kartograficznych. In: "Geografia Powszechna" Tom I. „Ziemia - środowisko naturalne człowieka”. Warszawa: PWN, pp. 102-120.

Van Leeuwen D., Strebe D., 2006, A Slice-and-Dice approach to area equivalence in polyhedral map projections. "Cartography and Geographic Information Science" Vol. 33, no. 4, pp. 269-286.

Yang H., Qureshi R., Sacan A., 2012, Protein surface representation and analysis by dimension reduction. "Proteome Science" 10 (Suppl. 1):S1, doi:10.1186/1477-5956-10-S1-S1

Zelnik-Manor L., Peters G., Perona P., 2005, Squaring the circle in panoramas. "Computer Vision" 2005. ICCV 2005. Tenth IEEE International Conference, Vol. 2, pp. 1292-1299.

\section{Internet sources}

www.learnnc.org/lp/editions/mapping/6434 